\title{
Dietary Cod Protein Improves the IGF1-Akt/PKB Signaling Pathway in Rat Skeletal Muscle during Recovery from Injury
}

\author{
Junio Dort ${ }^{1}$, Nadine Leblanc ${ }^{1}$, Claude H. Coté ${ }^{2}$, Hélène Jacques ${ }^{1 *}$ \\ ${ }^{1}$ School of Nutrition, Laval University, Institute of Nutraceuticals and Functional Foods, Laval University, Quebec Qc G1V 0A6, \\ Canada \\ ${ }^{2}$ Department of Rehabilitation, Laval University, University Hospital Centre, Laval University, Laurier Blvd, Quebec Qc G1V 4G2, \\ Canada \\ *Corresponding Author: Helene Jacques, School of Nutrition, Paul-Comtois Building, 2425 Agriculture Street, Laval Univer- \\ sity, Quebec, Canada. Tel: 418-656-2131, ext. 3864; Fax: 418-656-3353; E-mail: helene.jacques@fsaa.ulaval.ca
}

\begin{abstract}
The objective of this study was to identify molecular mechanisms underlying the beneficial effects of cod protein feeding on skeletal muscle recovery through detailed analyses of the IGF1-Akt/PKB signaling pathway. Acute mucle injury was induced by injecting bupivacaine in one tibialis anterior of Wistar rats fed isoenergetic diets containing either casein $(\mathrm{C})$, cod protein $(\mathrm{CP})$, or casein supplemented with a mixture of arginine, glycine, taurine and lysine $(\mathrm{C}+)$, matching their respective levels as in CP; downstream IGF1-Akt/PKB (protein kinase B) effectors were measured post-injury. Rats fed the $\mathrm{CP}$-diet exhibited a $50 \%$ reduction of the muscle ring finger1 (MuRF1) level compared with those fed the $\mathrm{C}$-diet $(\mathrm{p}=0.01)$, while the reduction induced by the $\mathrm{C}+$-diet was intermediary to, but did not differ significantly $(\mathrm{p}=0.07)$ from the $\mathrm{C}$-diet at day 2 post-injury. These findings indicate a decrease in the ubiquitination of muscle proteins and their degradation in the CP-group partly because of its high levels of arginine, glycine, taurine and lysine. Furthermore, phospho-Akt Ser473, a phosphorylation required for maximum activation of Akt, was increased in the CP-group at day $5(\mathrm{p}<0.0001)$ and day 28 post-injury $(\mathrm{p}=0.002)$ compared with $\mathrm{C}$, and at day 5 compared with $\mathrm{C}+(\mathrm{p}=0.0005)$. The phosphorylated form of GSK-3 was also increased with $\mathrm{CP}$ feeding at day 28 post-injury compared with $\mathrm{C}(\mathrm{p}=0.05)$, suggesting enhanced protein synthesis in the $\mathrm{CP}$ group. $\mathrm{C}+$ enhanced phospho-Akt Ser473 compared with $\mathrm{C}(\mathrm{p}=0.008)$ only at day 28 post-injury, with no effect on GSK-3 phosphorylation. Therefore, CP may reduce protein degradation induced by muscle injury through early modulation of the ubiquitination process, while enhancing muscle protein synthesis through Akt phosphorylation at later time points.
\end{abstract}

\section{Received Date: May 26, 2015 \\ Accepted Date: October 31, 2015 \\ Published Date: November 05, 2015}

Citation: Jacques, H., et al. Dietary Cod Protein Improves the IGF1-Akt/PKB Signaling Pathway in Rat Skeletal Muscle during Recovery from Injury. (2015) Int J Food Nutr Sci 2(2): 140-146.

Keywords: Cod protein; Injury; Muscle repair; Protein kinase B; Muscle ring finger1

DOI: $10.15436 / 2377-0619.15 .025$

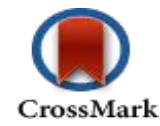

\section{Introduction}

Muscle mass maintenance relies on relative rates of protein synthesis and degradation. Positive mediators of protein synthesis include mechanical/chemical stimuli that increase IGF-1 signaling and meal-induced elevation in insulin, which both stimulate mammalian target of rapamycin (mTOR) through phosphoinositol-3 kinase (PI3K)/Akt activation ${ }^{[1-3]}$. Thereafter, mTOR activates the $70-\mathrm{kD}$ ribosomal S6 protein kinase (p70s6k) and the eukaryotic translation initiation factor (4E-eIF4E). PI3K/Akt can also integrate feedback from a variety of growth-related stimuli to regulate myofiber size by phosphorylating and thus repressing the glycogen synthase kinase-3 (GSK-3), resulting in a rapid release of eIF-2B and a concomitant increase in protein synthesis ${ }^{[4]}$. Conversely, negative mediators of protein degradation include insulin and IGF-1 both of which, by activating PI3K/Akt, phosphorylate and inactivate forkhead $\mathrm{O}(\mathrm{FoxO})$ factor/muscle ring finger1 (MuRF1) and atrogin-1, two important putative players in the atrophying process following injury ${ }^{[5]}$.

Injury can acutely induce insulin ${ }^{[6]}$ or insulin-like growth factor-1 (IGF-1) resistance ${ }^{[7]}$ and adversely affect skeletal muscle outcome by reducing protein synthesis while accelerating muscle protein catabolism, culminating in reduced fiber size, altered muscle mass recovery and loss of muscle function. Collectively, these data support the concept that strategies maintaining insulin or

Copy rights: (C2015 Jacques, H. This is an Open access article distributed under the terms of Creative Commons Attribution 4.0 International License. 
IGF-1 sensitivity are clearly required for efficient skeletal muscle repair following injury.

Cod protein has been shown to enhance insulin sensitivity ${ }^{[8,9]}$ as well as muscle mass recovery after an injury ${ }^{[10,11]}$. However the molecular mechanisms responsible for the effect of cod protein on muscle repair are still poorly understood. We first demonstrated that it was partly through modulation of inflammation and further showed that the anti-inflammatory properties of cod protein were driven by its high levels of arginine, glycine, taurine and lysine ${ }^{[11]}$. However, despite having similar effects on inflammation, a casein diet supplemented with a mixture of these amino acids to match their respective levels in cod protein did not allow optimal recovery in terms of muscle mass as did $\operatorname{cod}_{\text {protein }}{ }^{[11]}$. It remains unknown whether other crucial hypertrophic pathways such as the IGF-1/PI3K/Akt might underlie the beneficial effect seen with cod protein during the recovery period. The present study was thus conducted to gain further insight into 1) the mechanism behind the effects of cod protein and 2) the effect of supplementing casein with an amino acid mixture composed of arginine, glycine, taurine and lysine to achieve levels found in cod protein, on IGF-1-Akt regulating atrophic and hypertrophic effectors in injured skeletal muscle of the same rats previously used ${ }^{[11]}$. Because of the well-known insulin sensitizing effect of cod protein, we hypothesized that cod protein and casein enriched in arginine, glycine, taurine and lysine would result in 1) an increase in IGF-1-Akt signaling and 2) a decrease in atrogene expression in rat skeletal muscle following an injury. To test these hypotheses, the levels of IGF-1 and the total muscle protein content were evaluated in regenerating tibialis anterior muscles. We also performed western blot analysis on the protein levels of serine-phosphorylation of Akt; its downstream hypertrophic effectors (p70s6k, phosphorylated forms of GSK3 ), and its downstream regulated atrophic effectors atrogin-1 and MuRF1.

\section{Materials and Methods}

\section{Animals and Experimental Design}

Growing Wistar rats (50-60 g) were purchased from Charles River Laboratories (Saint-Constant, QC, Canada) and housed in individual plastic cages in a room maintained at $20^{\circ} \mathrm{C}$ ( $45-55 \%$ of humidity) with a $12 \mathrm{~h} / 12 \mathrm{~h}$ light-dark cycle. Following a 7-day adaptation period, the rats were randomly divided into three dietary groups. They were then gradually transferred to their respective experimental purified diet (ED), on which they were divided into five sub-groups according to the day of sacrifice $(0,2,5,14$ and 28 post-injury). Experimental diets and water were provided ad libitum. Treatment procedures and animal care were approved by the Laval University Animal Care Committee in accordance with the Canadian Council on Animal Care guidelines.

\section{Experimental Purified Diets}

The composition of the powdered purified diets was similar except for the protein source, which consisted of casein (C), cod protein (CP) or casein supplemented with a mixture of L-arginine $(0.45 \%)$, glycine $(0.43 \%)$, L-taurine $(0.17 \%)$ and L-lysine $(0.44 \%)(\mathrm{C}+)$. This amino acid mixture was added in order to reach similar amounts of these amino acids in both the $\mathrm{CP}$ and $\mathrm{C}+$ diets. The $\mathrm{CP}$ was prepared in our laboratory from frozen cod fillet as described elsewhere ${ }^{[10]}$. Arginine, glycine and taurine were added because of their anti-inflammatory properties. However, lysine was incorporated as an essential amino acid and to avoid any lysine-arginine imbalance. The level of protein in the purified diets was adjusted to an isonitrogenous basis at the expense of cornstarch and sucrose.

The formulation of the purified diets is shown in Table 1 and the amino acid composition of each protein used in the diets has been detailed previously ${ }^{[11]}$. All ingredients were supplied by MP Biomedicals (Solon, OH, Canada), except for lard and soybean oil, which were purchased from local supermarket. Butylated hydroxytoluene (BHT) was added to experimental diets in order to prevent oxidation of either n-6 (PUFA) in lard or n- 6 and n-3 (PUFA) in soybean oil, necessary to meet essential fatty acids requirement of rats $^{[12]}$. All of three purified diets were isoenergetic, isolipidic and isonitrogenous.

Table 1: Composition of the purified diets ( $g / 100 \mathrm{~g}$ of diet)

\begin{tabular}{|l|c|c|c|}
\hline & \multicolumn{3}{|c|}{ Dietary treatments } \\
\hline Ingredients $^{\prime}$ & $\mathrm{C}$ & $\mathrm{CP}$ & $\mathrm{C}^{+}$ \\
\hline Sucrose $^{1}$ & 20 & 20.5 & 20.3 \\
\hline Cellulose $^{1}$ & 5 & 5 & 5 \\
\hline Cornstarch $^{1}$ & 32.5 & 33 & 32.8 \\
\hline Casein $^{1,2}$ & 22.5 & - & 20.5 \\
\hline Cod protein $^{2,3}$ & - & 21.6 & - \\
\hline Arginine $^{1}$ & - & - & 0.45 \\
\hline Glycine $^{1}$ & - & - & 0.43 \\
\hline Lysine $^{1}$ & - & - & 0.44 \\
\hline Taurine $^{1}$ & - & - & 0.17 \\
\hline Cholesterol $^{1}$ & 1 & 1 & 1 \\
\hline Lard $^{4}$ & 10 & 10 & 10 \\
\hline Soya oil $^{5}$ & 4 & 4 & 4 \\
\hline Minerals $^{1,6}$ & 3.5 & 3.5 & 3.5 \\
\hline Vitamins $^{1,7}$ & 1 & 1 & 1 \\
\hline BHT $^{1}$ & 0.2 & 0.2 & 0.2 \\
\hline Choline bitartrate $^{1}$ & & 0.3 & 0.3 \\
\hline
\end{tabular}

$\mathrm{C}$, casein; $\mathrm{CP}$, cod protein $\mathrm{C}^{+}$, casein supplemented with arginine, glycine, taurine and lysine; BHT, butylated hydroxytoluene.

${ }^{1}$ Purchased from MP Biochemicals (Solon, Ontario, Canada)

${ }^{2}$ Casein, $89 \%$ protein; cod protein, $93 \%$ protein.

${ }^{3}$ Extracted from cod fillets purchased from local distributor (High Liner Foods Inc. Lachine, Canada).

${ }^{4}$ Purchased from local supermarket (Maple Leaf, Burlington, Canada).

${ }^{5}$ Purchased from local supermarket (Loblaws Inc., Toronto, Ontario, Canada).

${ }^{6} \mathrm{AIN}-93 \mathrm{G}$ purified mineral mix for laboratory rodents. AIN-93G mineral mix provides the following $(\mathrm{g} / 100 \mathrm{~g}$ mix): calcium carbonate, 35.7 ; monopotassium phosphate, 19.6; potassium citrate monohydrate, 7.078; sodium chloride, 7.4; potassium sulphate, 4.66; magnesium oxide, 2.4; ferric citrate, 0.606; zinc carbonate, 0.165 ; manganese carbonate, 0.063 ; copper carbonate, 0.03 ; potassium iodate, 0.001 ; sodium selenate anhydrous, 0.00103 ; ammonium molybdate $* 4 \mathrm{H}_{2} \mathrm{O}, 0.000795$; sodium metasilicate* $9 \mathrm{H}_{2} \mathrm{O}, 0.1454$; chromium potassium sulphate* $12 \mathrm{H}_{2} \mathrm{O}, 0.0275$; lithium chloride, 0.00174; boric acid, 0.008145; sodium fluoride, 0.00635 ; nickel carbonate, 0.00318; ammonium vanadate, 0.00066; powdered sugar, 22.1 .

${ }^{7} \mathrm{AIN}-93$ VX Vitamin Mix Fortification provides the following ( $\mathrm{g} / \mathrm{kg} \mathrm{mix}$ ): nicotinic acid, 3.0; D-calcium pantothenate, 1.6; pyridoxine hydrochloride, 0.7 ; thiamine hydrochloride, 0.6 ; riboflavin, 0.6 ; folic acid,

0.2 ; d-biotin, 0.02 ; vitamin $\mathrm{B}_{12}$ (0.1\% triturated in mannitol), 2.5 ; $\alpha$-tocopherol pow$\operatorname{der}(250 \mathrm{U} / \mathrm{g} ; 184 \mathrm{mg} / \mathrm{g}), 300.0$; vitamin A palmitate $(250000 \mathrm{U} / \mathrm{g} ; 137 \mathrm{mg} / \mathrm{g}), 1.6$; vitamin $\mathrm{D}_{3}(400000 \mathrm{U} / \mathrm{g} ; 10000 \mathrm{mg} / \mathrm{g}), 0.25$; phylloquinone, 0.075 ; powdered sucrose, 959.655 .

\section{Myotoxin Injury Protocol and Muscle Collection}

The procedure of muscle injury and collection used 
in this study is well described elsewhere ${ }^{[10]}$. After being fed experimental diets for 21 days, tibialis anterior (TA) muscles of both legs were removed from 8 rats per group under anesthesia with isoflurane $(2.5 \% / \mathrm{LO} 2)$. Muscle lysates were immediately prepared with lysis buffer and centrifuged at 10,000 g (10 min, $4^{\circ} \mathrm{C}$ ). The lysis buffer contained $20 \mathrm{mM}$ Tris, pH 7.5; $140 \mathrm{mM}$ $\mathrm{NaCl} ; 10 \mu \mathrm{M} \mathrm{MgCl} 2 ; 1 \mu \mathrm{M} \mathrm{CaCl} 2 ; 2 \mu \mathrm{M} \mathrm{NaF} ; 8.3 \mu \mathrm{M}$ Na3VO4; $2 \mu \mathrm{M}$ phenylmethylsulfonyl fluoride (PMSF); $1 \mathrm{~mL}$ glycerol; $100 \mu \mathrm{L}$ Igepal and $1 \%$ of protease inhibitors. The protein suspension was aliquoted and stored at $-80^{\circ} \mathrm{C}$ for future analysis. Animals were then sacrificed by exsanguination.

Thirty-two animals per group were then anesthetized with isoflurane $(2.5 \% / \mathrm{LO} 2)$ and given buprenorphine $(0.1 \mathrm{mg} /$ $\mathrm{kg}, \mathrm{sc}$ ), after which one TA muscle of each animal was injured using $200 \mu \mathrm{l}$ of bupivacaine (Marcaine, Abbott, Mississauga, Ontario, Canada) while the contra-lateral muscle was injected with a similar volume of saline and served as sham. Rats were returned to their respective cages and both TA muscles were removed ( 8 animals per dietary group/time point) at days $2,5,14$ and 28 post-injury, and processed as described above. As previously indicated, time points were chosen to examine the time course of inflammation and regeneration in bupivacaine-induced acute muscle damage in terms of the effect exerted by protein feeding on biochemical aspects corresponding to the acute catabolic phase (day 2), to the initiation of regeneration (day 5), to progressive (day 14) and complete (day 28) muscle regeneration $^{[10,11]}$. Given that atrogin-1 and MuRF1 are significantly up-regulated as early as $2-5$ days post-trauma ${ }^{[13]}$, these proteins were quantified only at these time points. The bupivacaine did not cause any side effect on general health status of rats and additional doses of buprenorphine were given twice daily until the third day post-injury.

\section{ELISA and BCA Assays}

ELISA was performed to determine the level of IGF-1 in the lysate of injured muscle using the kit purchased from R\&D Systems (MG100; R\&D Systems, Minneapolis, MN, USA) and the protocol provided by the manufacturer. The muscle protein content was quantified using the BCA Assay Kit (Thermo scientific, Mississauga, ON, Canada), according to the manufacturer protocol.

\section{Western Blot Assay}

Fifty $\mu \mathrm{g}$ of protein were diluted in sample buffer and boiled for $2 \mathrm{~min}$. The protein samples were electrophoretically separated on a 9\%-10\% SDS-polyacrylamide gel and electrotransferred to Immobilon-P Transfer Membranes, except for atrogin-1 which was transferred to polyvinylidene difluoride (PVDF) membranes. Membranes were stained with Ponseau S to confirm the transfer of protein, after which they were serially washed with buffer and blocked with BSA (5\%) for $2 \mathrm{~h}$. Membranes were then blocked overnight with either the rabbit polyclonal anti-phospho-Akt (Ser473), Akt, phospho-P70S6K, phospho-GSK-3, GSK-3 (Cell Signaling Technology Inc., Trask Lane Danvers, MA, USA), or MuRF1 (Abcam Inc., Toronto, ON, Canada), all diluted in BSA 5\% (1:1000). For atrogin-1, membranes were blocked for $1 \mathrm{~h}$ at room temperature with the rabbit polyclonal anti-atrogin-1 (ECM Biosciences, Versailles, KY, USA), diluted in 5\% non-fat milk (1:1000). GAPDH (Santa Cruz Biotechnology, Santa Cruz, CA) was used as a protein load- ing control. After rinsing, the membranes were incubated for $1 \mathrm{~h}$ with anti-rabbit IgG polyclonal secondary antibody (1/3000) and bands were revealed using ECL-plus Western blotting reagent (PerkinElmer Life and Analytical Sciences, Wellesley, MA) according to manufacturer's instructions. The signal intensities were captured (Fusion FX7, Montreal Biotech Inc., Montreal, Canada), corrected for the local background and quantified using the BIO-1D advanced software (Montreal Biotech Inc., Montreal, Canada). Results were normalized relative to respective GAPDH intensities.

\section{Statistical Analysis}

Data were analysed with the MIXED procedure of the Statistical Analysis System (SAS Institute, version 9.2, Cary, NC, USA). Normality was verified according to the Shapiro-Wilk test. Data for MuRF1 were then log-transformed before statistical analysis. Dietary protein effects were determined by means of an analysis of variance (ANOVA) and Fisher's protected LSD test when a significant $F$ ratio was observed. All values are expressed as the mean \pm SEM. P values $\leq 0.05$ were considered statistically significant.

\section{Results}

Food intake throughout the study period and initial body weight did not differ between experimental groups (Table 2). However, daily CP ingestion increased body weight gain compared to either $\mathrm{C}(\mathrm{p}=0.05)$ or $\mathrm{C}+(\mathrm{p}=0.04)$ at day 28 post-injury and this was associated with larger muscle fiber CSA.

Table 2: Food intake and body weight gain of each sub-group of rats sacrificed at different time points.

\begin{tabular}{|c|c|c|c|c|}
\hline Parameters & Days post- injury & Casein & Cod protein & Casein $^{+}$ \\
\hline \multirow{4}{*}{$\begin{array}{c}\text { Mean food } \\
\text { intake } \\
\text { (g/d) }\end{array}$} & 0 & $22.3 \pm 0.6$ & $22.8 \pm 1.2$ & $22.9 \pm 1.0$ \\
\cline { 2 - 5 } & 2 & $21.6 \pm 0.3$ & $22.3 \pm 0.6$ & $23.3 \pm 0.9$ \\
\cline { 2 - 5 } & 14 & $22.6 \pm 0.9$ & $23.2 \pm 0.8$ & $22.4 \pm 0.3$ \\
\cline { 2 - 5 } & 28 & $22.9 \pm 0.8$ & $23.6 \pm 0.6$ & $23.9 \pm 0.8$ \\
\hline \multirow{4}{*}{$\begin{array}{c}\text { Initial } \\
\text { body }\end{array}$} & 0 & $152 \pm 3$ & $154 \pm 3$ & $153 \pm 6.4$ \\
\cline { 2 - 5 } weight \\
\cline { 2 - 5 }$(\mathrm{g})$
\end{tabular}

Values are mean $\pm \operatorname{SEM}(\mathrm{n}=7-8$ rats/diet/time point $)$.

$a, b, c$ Values on the same line with the same letter are not significantly different $(\mathrm{p} \leq 0.05)$. $\uparrow$ Histomorphometry obtained from sections stained with hematoxylin and eosin as previously reported in the same animals ${ }^{[1]}$. Values are provided for the injured muscle across time point. 
Figure 1 shows the concentration of IGF-1 in injured muscle at days $0,2,5,14$ and 28 post-injury. After an initial rise at day 2 post-injury, the amount of this protein diminished progressively over time but it was not altered in response to protein feeding. However, as noted in Figure 2, CP significantly increased Ser473-phosphorylation of Akt compared to C $(\mathrm{p}<0.0001)$ and $\mathrm{C}+(\mathrm{p}=0.0005)$ at day 5 post-injury while total Akt content remained unchanged. At the same time point, Ser473-phosphorylation of Akt in the C+ group did not significantly different from that of the $\mathrm{C}(\mathrm{p}=0.62)$. At day 28 post-injury, Ser473-phosphorylation of Akt was increased in both CP$(p=0.002)$ and $\mathrm{C}+-(\mathrm{p}=0.0008)$ fed groups compared with their $\mathrm{C}$-fed counterparts.

Changes in the phosphorylation of hypertrophic effectors are shown in Figures 3 and 4 . The effect of $\mathrm{CP}$ was visible only at day 28 post-injury when it enhanced the phosphorylation of GSK3 on Ser2 1 compared with $\mathrm{C}(\mathrm{p}=0.05)$. The $\mathrm{C}+\operatorname{diet}$ provoked an intermediate response which was not different from that induced by the $\mathrm{C}(\mathrm{p}=0.31)$ or $\mathrm{CP}(\mathrm{p}=0.57)$ diets (Figure $3 \mathrm{~A})$. The diets had no effect on either Ser9-phosphorylation of GSK3 (Figure 3B) or phosphorylation of p70s6k at any studied time point (Figure 4).

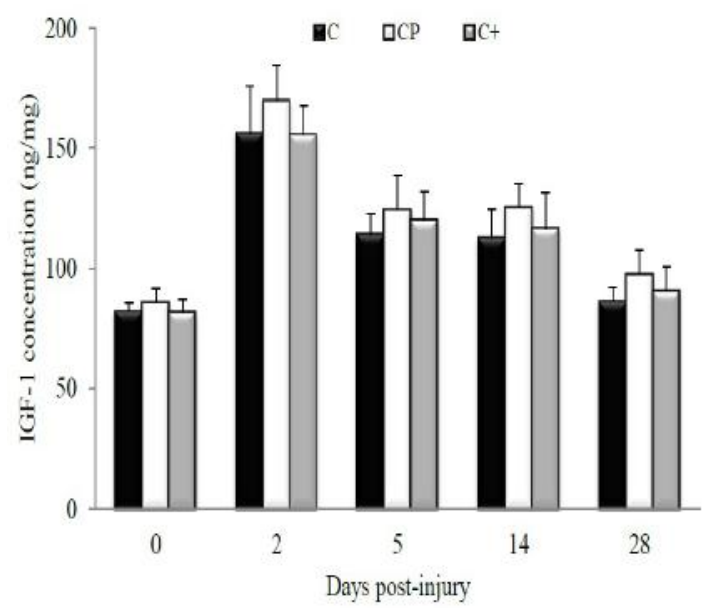

Figure 1: Changes in muscle IGF-1 concentration in rats fed either casein $(\mathrm{C})$, cod protein $(\mathrm{CP})$ or casein supplemented with L-arginine, glycine, L-taurine, and L-lysine $\left(\mathrm{C}^{+}\right)$.Values are mean \pm SEM $(n=7-8$ rats/diet at every time point). ELISA analysis showed that the level of IGF-1 was increased in the injured muscles regardless of the group, with a peak at day 2 post-injury, at which time point the effect of diets on muscle IGF-1 might be diluted. $\mathrm{p} \leq 0.05$.
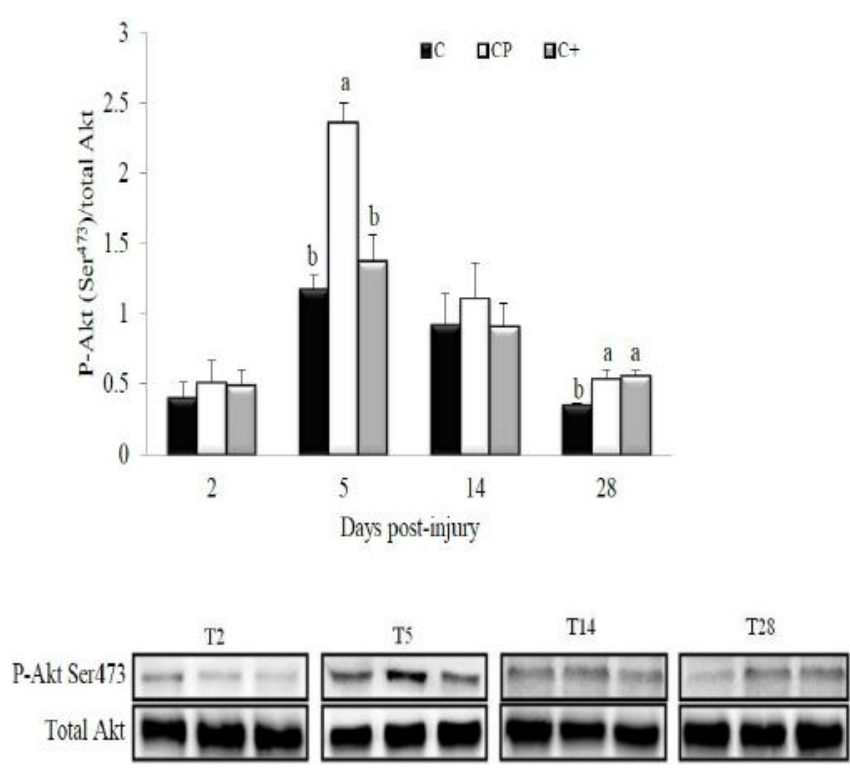

Figure 2: Effect of dietary proteins on Ser473-phosphorylation of Akt in regenerating tibialis anterior muscle of rats at days 2, 5, 14 and 28 post-injury. A representative Western blot is displayed in the lower panel for each studied time point. Values are mean \pm SEM $(n=7-8$ rats/diet at every time point). Results are expressed relative to values obtained at day 0 . Groups bearing different letters for a given time point are significantly different $(\mathrm{p} \leq 0.05)$. $\mathrm{C}$, casein; $\mathrm{CP}$, cod protein; $\mathrm{C}+$, casein supplemented with L-arginine, glycine, L-taurine and L-lysine.
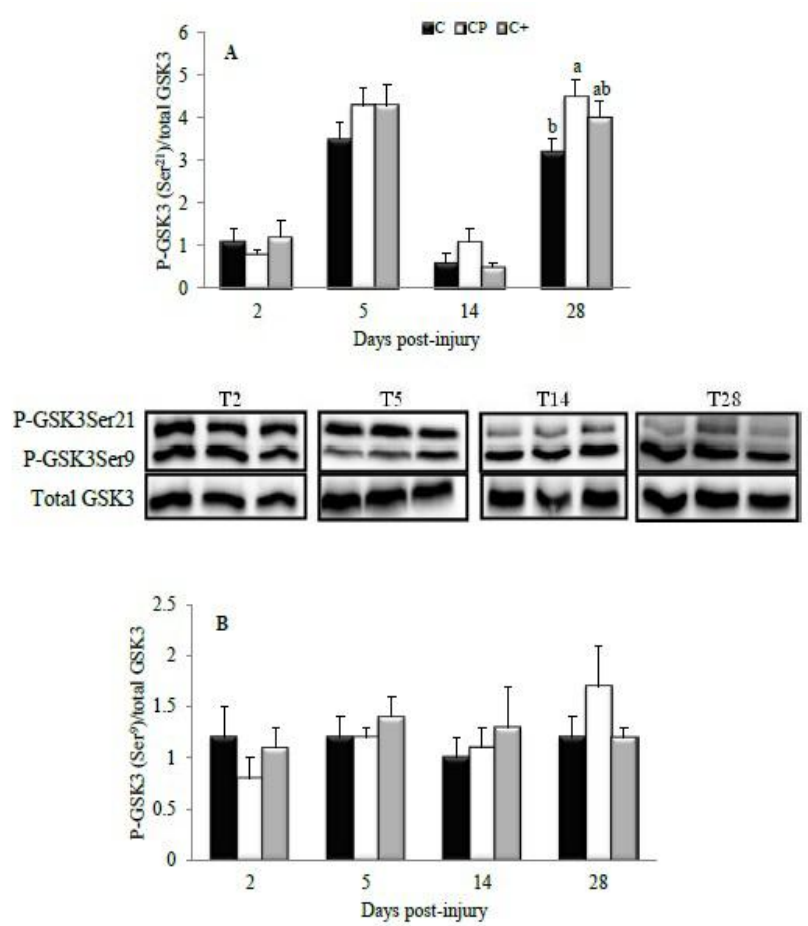

Figure 3: Effect of dietary proteins on GSK3 phosphorylation in rat tibialis anterior muscle during recovery from injury. A representative Western blot is provided for both Ser21- (A) and Ser9-phosphorylation of GSK3 (B). Values are mean \pm SEM $(n=7-8$ rats/diet at every time point). All values were corrected for GAPDH as a protein loading control. Results are expressed relative to values obtained at day 0 . Groups bearing different letters for a given time point are significantly different $(\mathrm{p} \leq 0.05)$. $\mathrm{C}$, casein; $\mathrm{CP}$, cod protein; $\mathrm{C}+$, casein supplemented with L-arginine, glycine, L-taurine and L-lysine. 

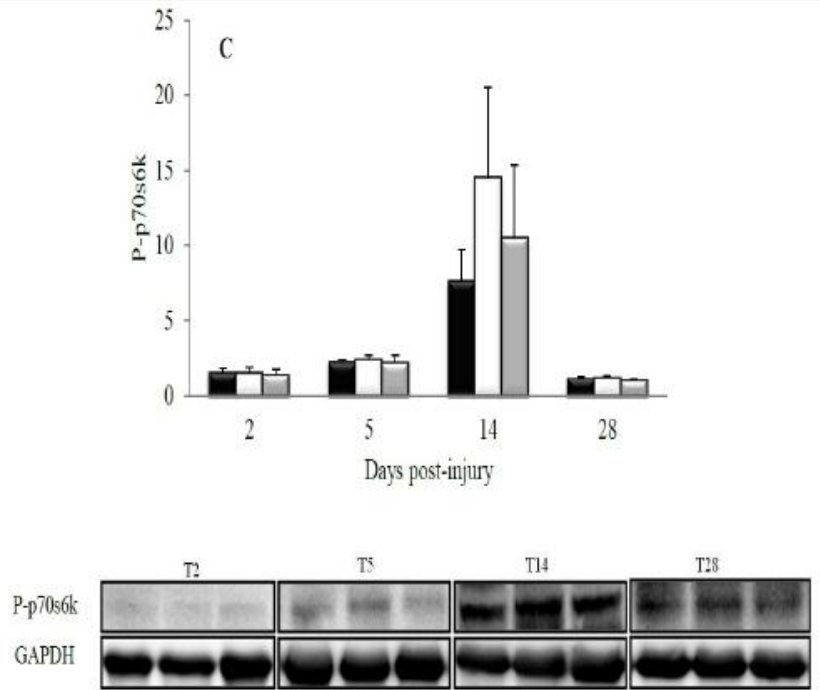

Figure 4: Effect of dietary proteins on the phosphorylation of p70s6k in rat tibialis anterior muscle during recovery from injury. A representative Western blot is displayed at the lower panel for each studied time point. Values are mean \pm SEM $(n=7-8$ rats/diet at every time point). All values were corrected for GAPDH as a protein loading control. C, casein; CP, cod protein; $\mathrm{C}+$, casein supplemented with L-arginine, glycine, L-taurine and L-lysine.

Figure 5 shows the effects of dietary proteins on the levels of atrophic effectors early after muscle injury (day 2 and day 5 post-injury). Interestingly, the $\mathrm{CP}$ diet significantly $(\mathrm{p}=0.01)$ reduced by $50 \%$ the level of MuRF1 compared with the $\mathrm{C}$ diet at day 2 post-injury, while the $\mathrm{C}+$ diet tended to reduce the level of MuRF1 by $38 \%$ compared with the $\mathrm{C}$ diet $(\mathrm{p}=0.07)$ (Figure $5 \mathrm{~A}$ ). No significant changes in the level of atrogin-1 were observed between the dietary proteins (day $2, p=0.17$; day $5, p=0.60$ ) (Figure 5B).

$$
\text { A }
$$
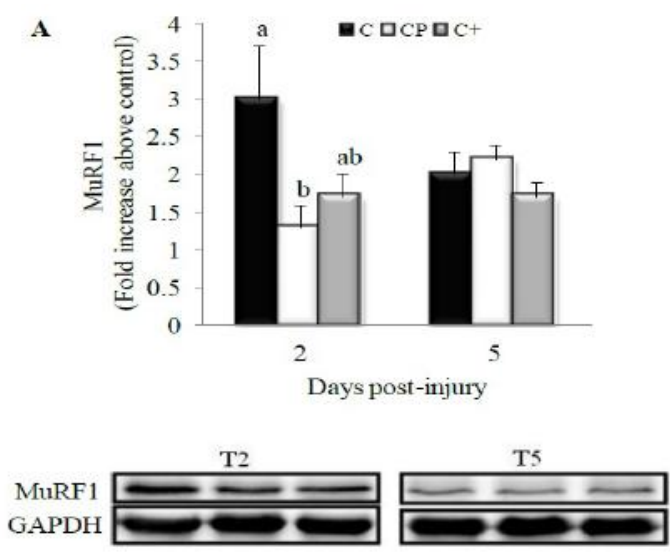

Figure 5A: Effect of dietary proteins on the levels of atrophic proteins in rat tibialis anterior muscle during recovery from injury. A representative Western blot is displayed in the lower panel for either MuRF1 (A) or atrogin-1
B
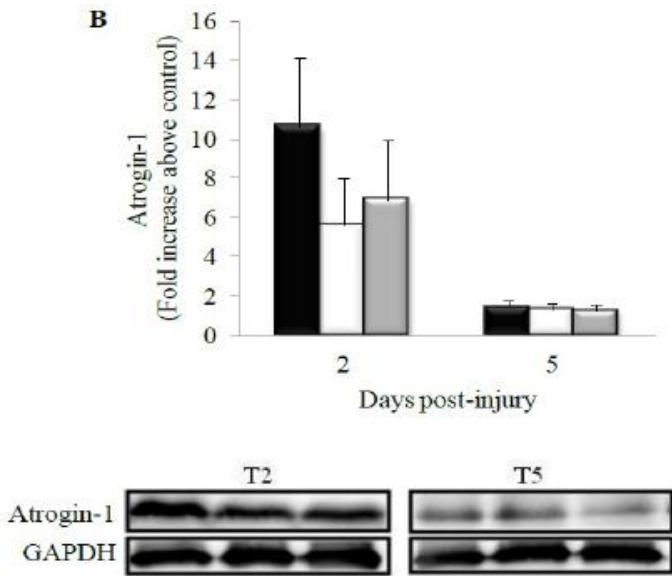

Figure 5B: Values are mean \pm SEM $(n=7-8$ rats/diet at every time point). All values were corrected for GAPDH as a protein loading control. Results are expressed relative to values obtained at day 0 . Groups bearing different letters for a given time point are significantly different $(\mathrm{p} \leq 0.05)$. MuRF1 at day 2 post-injury: $\mathrm{p}=0.07$ for $\mathrm{C}+\mathrm{vs} \mathrm{C}$; $\mathrm{C}$, casein; $\mathrm{CP}$, cod protein; $\mathrm{C}+$, casein supplemented with $\mathrm{L}$-arginine, glycine, L-taurine and L-lysine.

Higher total muscle protein content was observed in both the $\mathrm{CP}$ - and $\mathrm{C}+$-fed rats compared with the $\mathrm{C}$-fed counterparts at day 5 post-injury $(\mathrm{C}$ vs $\mathrm{CP}, \mathrm{p}=0.03$; $\mathrm{C}$ vs $\mathrm{C}+, \mathrm{p}=0.02$ ) (Figure 6). At day 14 post-injury, $\mathrm{CP}$ also increased muscle protein content compared with $\mathrm{C}(\mathrm{p}=0.05)$. Muscle protein level of the $\mathrm{C}+$-fed rats did not differ from that of the $\mathrm{C}-(\mathrm{p}=0.30)$ and $\mathrm{CP}$-fed rats $(\mathrm{p}=0.57)$. However, at day 28 post-injury, the $\mathrm{CP}$ diet increased by $11 \%$ muscle protein content compared with the $\mathrm{C}$ and $\mathrm{C}+$ diets without reaching the level of significance.

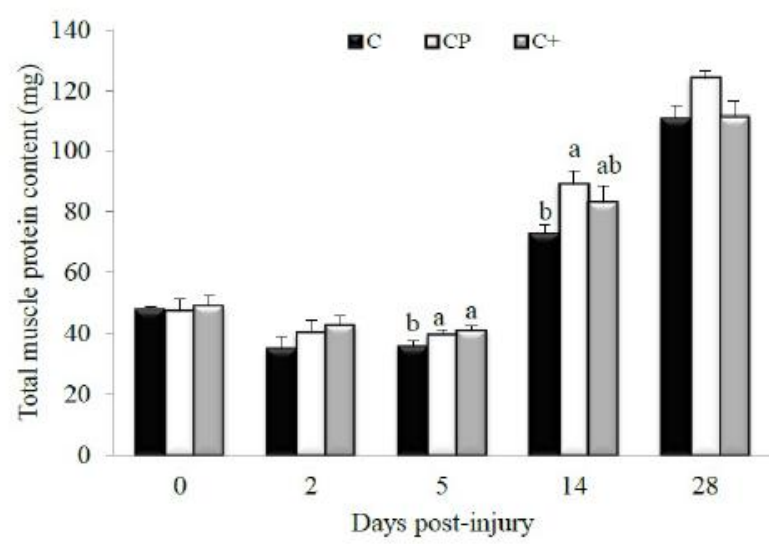

Figure 6: Effect of dietary proteins on whole muscle protein content in regenerating rat tibialis anterior muscle measured by BCA Protein Assay Kits. Values are mean \pm SEM ( $n=7-8$ rats/diet at every time point). Groups bearing different letters for a given time point are significantly different $(\mathrm{p} \leq 0.05)$. $\mathrm{C}$, casein; $\mathrm{CP}$, cod protein; $\mathrm{C}+$, casein supplemented with L-arginine, glycine, L-taurine and L-lysine.

\section{Discussion}

This study aimed at getting further insight into the molecular mechanisms involved in the beneficial effect of cod protein on skeletal muscle recovery following injury. The main results show that 1) $\mathrm{CP}$ decreased MuRF1 at day 2 post-injury while increasing the level of phospho-Akt Ser473 at day 5 
and of both phospho-Akt Ser473 and phospho-GSK-3 at day 28 post-injury, and 2) these effects appear to be partly attributed to the high levels of arginine, glycine, taurine and lysine in $\mathrm{CP}$, an interpretation based on the strong tendency observed with $\mathrm{C}+$ to reduce MuRF1 at day 2 post-injury and their increasing effects on phospho-Akt Ser473 at day 28 post-injury. These findings suggest that $\mathrm{CP}$ might be effective to improve IGF1-Akt signaling and to reduce atrogene expression in rat skeletal muscle during recovery from injury.

Previous studies in our lab have shown that CP-fed animals exhibited greater growth and regeneration of skeletal muscle after trauma, partly because of improved modulation of inflammation $^{[10,11]}$. In this respect, CP decreased the accumulation of pro-inflammatory ED1+-cells and COX-2 level while increasing the number of anti-inflammatory ED2+-cells in skeletal muscle during recovery from injury ${ }^{[11]}$. The novel aspect depicted with the current study is that, in addition to reducing pro-inflammatory cells at the site of injury, $\mathrm{CP}$ did protect against the upregulation of MuRF1-encoding E3 ubiquitin ligases at day 2 post-injury compared with $\mathrm{C}$, a finding in line with our second hypothesis. Thus, the anti-catabolic effect of CP could not only be related to a blunted inflammatory reaction but also associated with attenuation of atrogene expression, resulting in larger fiber cross-sectional area in animals fed the CP diet at day 28 (Table 2). As part of the ubiquitin-proteasome system required for myofiber protein degradation such as myosin and myogenin, MuRF1 expression can be controlled by PI3K/Akt-driven signals ${ }^{[14]}$. However, the lack of an increased Akt-phosphorylation at day 2 post-injury suggests that the beneficial effect of CP on reducing MuRF1-atrophying program appears to be independent from the IGF-1/PI3K/Akt pathway. As reviewed elsewhere ${ }^{[13]}$, there could be an alternative unidentified inhibitory signaling pathway unrelated to the PI3K-Akt that might regulate the level of MuRF1 protein; for instance, activation of vital inflammatory mediators such as NF- $\kappa \mathrm{B}$ can enhance muscle proteolysis by increasing MuRF1 activity ${ }^{[13]}$. CP might thus down-regulate major inflammation-mediated catabolic events such as TNF- $\alpha$ through reducing pro-inflammatory cells, thereby improving the growth and recovery of muscle fibers. Growing evidence from our lab and elsewhere have confirmed the anti-inflammatory properties of fish proteins, demonstrated by a decrease of TNF- $\alpha$ in cultured human macrophages ${ }^{[15]}$, and of both TNF- $\alpha$ and interleukin- 6 expression in adipose tissue of high-fat/high-sucrose-fed rats $^{[16]}$. Whether decreased pro-inflammatory cytokines translate into inhibition of the NF- $\kappa \mathrm{B}$ pathway in muscle of CP-fed rats will require further investigation.

We previously observed that the beneficial effect of CP on inflammatory cell accumulation in rat skeletal muscle after injury are driven by its high levels of arginine, glycine, taurine and lysine ${ }^{[11]}$. However, this anti-inflammatory action could only partially explain the positive effect seen with CP on muscle recovery because the addition of such amino acids to casein did not support fiber growth and regeneration as did CP (Table 2). One key explanation is that the amino acid mixture partially reproduced the effect of CP on MuRF1. However signaling pathways for atrophy regulation by amino acids are still poorly understood. A recent study in H4-II-E cells showed suppressive effect of an amino acid mixture composed of Ala, Gln, His, Leu, Met, Pro, Trp, and Tyr on autophagy concomitant with reduced ROS production ${ }^{[17]}$. Moreover, although a non regulatory ami- no acid mixture that included Arg, Asn, Asp, Cys, Glu, Gly, Ile, Lys, Phe, Ser, Thr and Val had no effect on autophagy, individual amino acids such as Met, Arg, and Cys suppressed autophagy, as revealed by a drastic decrease of cytosolic LC3 ratio ${ }^{[17]}$. As arginine-regulated autophagy is independent from mTOR pathways ${ }^{[17]}$, it is also likely that glycine and taurine may decrease protein degradation through signal molecules other than PI3K-Akt that might negatively regulate MuRF1 expression in our study. Several studies have shown that ROS and pro-inflammatory cytokines mediate atrogene-induced autophagy ${ }^{[8,17]}$; arginine, glycine and taurine might thus exert a negative feedback loop on MuRF1 through their anti-oxidative and anti-inflammatory properties ${ }^{[11]}$. Lysine, one of the essential amino acids found in high amount in $\mathrm{CP}$, might play a multifaceted role in the outcome of fiber growth and recovery by stimulating protein synthesis as well as suppressing myofibrillar protein degradation through the autophagic-lysosomal system ${ }^{[18]}$. Collectively, our findings support the concept that the anti-catabolic action of $\mathrm{CP}$ in post-damage skeletal muscle was only in part attributed to its high levels of arginine, glycine, taurine and lysine. Further studies are needed to clarify the molecular mechanism through which other essential amino acids such as threonine, methionine and cysteine can mediate their suppressive effect on the autophagic-lysosomal system.

Our data regarding the marked up-regulation of muscle IGF-1 at day 2 post-injury is a consistent finding following myotoxic injury ${ }^{[19]}$. More importantly, although the level of IGF-1 within the injured muscle was not affected by protein feeding, CP might have exerted an insulin or IGF-1-sensitizing effect in line with our first hypothesis when it enhanced phospho-Akt Ser473 at days 5 and 28 post-injury compared with C. This was associated with an increased phospho-GSK-3 at day 28 post-injury. In support of these findings, it has already been shown that the consumption of cod protein compared to either casein or soy protein completely prevented the development of skeletal muscle insulin resistance by restoring insulin-induced activation of PI3K/Akt and increasing GSK-3 phosphorylation in rats fed a high fat diet ${ }^{[8,9]}$. Given that the repressor of protein synthesis, GSK-3, becomes inactive upon phosphorylation, and that CP increased phospho-GSK-3 compared with $\mathrm{C}$ at day 28 after injury, one can assume that $\mathrm{CP}$ might have increased protein synthesis during the recovery period, explaining thus the $11 \%$ increase in the muscle protein content at day 28 post-injury observed with CP.

Jacques, et al. ${ }^{[20]}$ examined the effect of various dietary protein sources on body composition of rats fed the same purified diets as in the present study during a 28-day period, and reported that $\mathrm{CP}$ increased body «protein:fat» ratio compared with casein. This observed beneficial effect of $\mathrm{CP}$ might also result, at least in part, from a direct action of amino acids on insulin and/or IGF-1 signaling in skeletal muscle cells. In this respect, previous data from our lab showed that L6 myocytes exposed to CP-derived amino acids exhibited greater rates of insulin-stimulated glucose uptake compared with cells incubated with casein-derived amino acids ${ }^{[8]}$. However, in the present study, the impact of the tested amino acid mixture on the hypertrophic potential during muscle recovery was partial. Indeed, although the amino acid mixture increased phospho-Akt Ser473 similarly to $\mathrm{CP}$ at day 28 post-injury, it was without an effect on the phosphorylated form of GSK-3 at this time point. It is likely 
that lower levels of essential amino acids such as methionine and threonine in $\mathrm{C}$ and $\mathrm{C}+$ might account for their reduced anabolic and/or anti-catabolic response compared with CP. Moreover from our data, it appears that Akt phosphorylation induced by $\mathrm{CP}$ has not been sufficient to activate their downstream effector p70S6K. As observed in age-related atrophy ${ }^{[21]}$, it is possible that injury causes a blockage between Akt and some downstream effectors, including p70S6K. In addition, measuring mTOR and IGF-1R or IRS-1 activation would help clarifying the effect of $\mathrm{CP}$ on IGF-1/Akt signaling during muscle recovery.

This investigation has the merit of studying several molecular effectors that are associated with improved muscle fiber recovery in $\mathrm{CP}$-fed rats compared with $\mathrm{C}$-fed counterparts.

\section{Conclusion}

Overall, we conclude that $\mathrm{CP}$ 1) may reduce muscle protein degradation through modulation of the ubiquitination process early after injury, and 2) upregulate protein synthesis through inactivation of GSK-3 at the later phase of recovery. Furthermore, we observed that the anti-catabolic and hypertrophic actions of CP during recovery from injury are partly driven by its high levels of arginine, glycine, taurine and lysine. In order to get a more complete understanding of the beneficial role of cod protein on muscle repair after an injury, studies on the influence of casein supplemented with sulfur amino acids and threonine in addition to arginine, glycine, taurine and lysine on post-damage muscle repair should be conducted.

\section{Acknowledgement}

We are grateful to the Natural Sciences and Engineering Research Council of Canada for financial support (HJ), to the Organization of American States for a two years fellowship granted to JD, and to Christelle Brèque and Christian Lessard from Dr. Janice Bailey’s laboratory for technical assistance.

\section{Conflict of Interest}

The authors declare no conflict of interest.

\section{References}

1. Anthony, J.C., Lang, C.H., Crozier, S.J., et al. Contribution of insulin to the translational control of protein synthesis in skeletal muscle by leucine. (2002) Am J Physiol Endocrinol Metab 282(5): E1092-E1101. 2. Zanchi, N.E., Lancha, A.H. Mechanical stimuli of skeletal muscle: implications on mTOR/p70s6k and protein synthesis. (2008) Eur J Appl Physiol 102(3): 253-263.

3. Wilson, G.J., Moulton, C.J., Garlick, P.J., et al. Post-meal responses of elongation factor 2 (eEF2) and adenosine monophosphate-activated protein kinase (AMPK) to leucine and carbohydrate supplements for regulating protein synthesis duration and energy homeostasis in rat skeletal muscle. (2012) Nutrients 4(11): 1723-1739.

Online ISSN: 2377-0619

Journal Title: International Journal Food and Nutritional Science Journal Short Name: Int J Food Nutr Sci
4. Doucet, M., Russell, A.P., Leger, B., et al. Muscle atrophy and hypertrophy signaling in patients with chronic obstructive pulmonary disease. (2007) Am J Respir Crit Care Med 176(3): 261-269.

5. Schiaffino, S., Mammucari, C. Regulation of skeletal muscle growth by the IGF1-Akt/PKB pathway: insights from genetic models. (2011) Skelet Muscle 1(1): 4.

6. Asp, S., Richter, E.A. Decreased insulin action on muscle glucose transport after eccentric contractions in rats. (1996) Journal of Applied Physiology 81(5): 1924-1928.

7. Chen, Y., Sood, S., Krishnamurthy, V.M.R. Endotoxin-induced growth hormone resistance in skeletal muscle. (2009) Endocrinology 150(8): 3620-3626.

8. Lavigne, C., Tremblay, F., Asselin, G., et al. Prevention of skeletal muscle insulin resistance by dietary cod protein in high fat-fed rats. (2001) Am J Physiol Endocrinol Metab 281(1): E62-E71.

9. Tremblay, F., Lavigne, C., Jacques, H., et al. Dietary cod protein restores insulin-induced activation of phosphatidylinositol 3-kinase/Akt and GLUT4 translocation to the T-tubules in skeletal muscle of highfat-fed obese rats. (2003) Diabetes 52(1): 29-37.

10. Dort, J., Sirois, A., Leblanc, N., et al. Beneficial effects of cod protein on skeletal muscle repair following injury.(2012) Appl Physiol Nutr Metab 37(3): 489-498.

11. Dort, J., Leblanc, N., Maltais-Giguere, J., et al. Beneficial effects of cod protein on inflammatory cell accumulation in rat skeletal muscle after injury are driven by its high levels of arginine, glycine, taurine and lysine. (2013) PLoS One 8(10): e77274.

12. Reeves, P.G., Nielsen, F.H., Fahey, G.J. AIN-93 purified diets for laboratory rodents: final report of the American Institute of Nutrition ad hoc writing committee on the reformulation of the AIN-76A rodent diet. (1993) J Nutr 123(11): 1939-1951.

13. Foletta, V.C., White, L.J., Larsen, A.E., et al. The role and regulation of MAFbx/atrogin-1 and MuRF1 in skeletal muscle atrophy. (2011) Pflugers Arch 461(3): 325-335.

14. Witt, S.H., Granzier, H., Witt, C.C., et al. MURF-1 and MURF-2 target a specific subset of myofibrillar proteins redundantly: towards understanding MURF-dependent muscle ubiquitination. (2005) J Mol Biol 350(4): 713-722.

15. Rudkowska, I., Marcotte, B., Pilon, G., et al. Fish nutrients decrease expression levels of tumor necrosis factor-alpha in cultured human macrophages. (2010) Physiol Genomics 40(3): 189-194.

16. Pilon, G., Ruzzin, J., Rioux, L.E., et al. Differential effects of various fish proteins in altering body weight, adiposity, inflammatory status, and insulin sensitivity in high-fat-fed rats. (2011) Metabolism 60(8): 1122-1130.

17. Angcajas, A.B., Hirai, N., Kaneshiro, K., et al. Diversity of amino acid signaling pathways on autophagy regulation: a novel pathway for arginine. (2014) Biochem Biophys Res Commun 446(1): 8-14.

18. Sato, T., Ito, Y., Nagasawa, T. Lysine suppresses myofibrillar protein degradation by regulating the autophagic-lysosomal system through phosphorylation of Akt in C2C12 cells. (2014) Springerplus 3: 584.

19. Lu, H.Y., Huang, D.P., Saederup, N., et al. Macrophages recruited via CCR2 produce insulin-like growth factor-1 to repair acute skeletal muscle injury. (2011) Faseb J 25(1): 358-369.

20. Jacques, H., Leblanc, N., Papineau, R., et al. Peanut protein reduces body protein mass and alters skeletal muscle contractile properties and lipid metabolism in rats. (2010) Br J Nutr 103(9): 1331-1339.

21. Li, M., Li, C., Parkhouse, W.S. Age-related differences in the IGF-I-mediated activation of Akt-1 and p70 S6K in mouse skeletal muscle. (2003) Mech Ageing Dev 124(7): 771-778. 